\title{
Mechanical Circulatory Support in ST-Elevation Myocardial Infarction
}

\author{
Nathan Lo and E. Magnus Ohman
}

\subsection{Introduction}

Cardiogenic shock (CS) occurs in approximately 8-10\% of patients with ST-elevation myocardial infarction (STEMI). While immediate percutaneous coronary intervention (PCI) continues to be the mainline treatment strategy, mortality remains as high as $40 \%$ before hospital discharge. Even after hospital discharge, survivors of cardiogenic shock complicating myocardial infarction (CSMI) often suffer from severe heart failure and its consequences, including repeated hospitalization and high mortality. Mechanical circulatory support (MCS) devices during CSMI PCI can be lifesaving by supporting poor ventricular function while decreasing myocardial wall stress and relieving ischemia. In this chapter, we review the definition and pathophysiology of CSMI, the major ventricular support devices, an algorithm for MCS use in STEMI, relevant data, and a prototypic case that illustrates CSMI management augmented by MCS.

\subsection{Definition and Identification of Cardiogenic Shock}

Cardiogenic shock is typically defined as a cardiac index $<2.2 \mathrm{~L} / \mathrm{min} / \mathrm{m}^{2}$ and systolic blood pressure $<90 \mathrm{mmHg}$ with signs of impaired organ perfusion such as decreased urine output, altered mental status, or cool extremities. Initial evaluation of a patient with STEMI suspected to be in CS should rely on clinical bedside evaluation (Table 19.1). Generally, the focused evaluation should determine clinical stability and whether the patient exhibits signs of low cardiac

\footnotetext{
N. Lo $\cdot$ E. Magnus Ohman $(\bowtie)$

Duke University Medical Center, Duke Clinical Research Institute, Durham, NC, USA

e-mail: erik.ohman@duke.edu
} 
Table 19.1 Important physical examination findings in patients with ST-elevation myocardial infarction suspected to be in cardiogenic shock

\begin{tabular}{l|l}
\hline Intravascular volume status_-wet" & Cardiac output_-cold" \\
\hline$\uparrow$ Jugular venous pressure & Cool extremities \\
\hline Rales & Hypotension \\
\hline $\mathrm{S}_{3}$ gallop & Narrow pulse pressure \\
\hline Orthopnea & Altered mental status
\end{tabular}

output and high intravascular volume. The Killip classification score combines assessment of cardiac output and intravascular volume and can be helpful to quickly assess patients suffering from heart failure after myocardial infarction. Killip Class I patients show no signs of heart failure; Class II patients have rales, $\mathrm{S}_{3}$ gallop, and venous hypertension; Class III patients have severe heart failure with pulmonary edema; and Class IV patients are in cardiogenic shock with hypotension and evidence of poor perfusion. Higher Killip score has been shown to be an independent predictor of mortality in STEMI patients and may be useful for quick risk stratification in the emergency department in patients presenting with acute coronary syndrome. This initial evaluation is vital as it guides further therapy and whether vasopressors, inotropes, or MCS need to be started concurrent with or even prior to PCI.

\subsection{Pathophysiology of Cardiogenic Shock Complicating Myocardial Infarction}

CS begins with pump failure but soon becomes systemic, multiorgan failure. Pump failure begins a "downward spiral" which-if unchecked-potentiates itself as worsened ischemia, causing progressive myocardial dysfunction and eventually death (Fig. 19.1). Pump failure leads to decreased cardiac output, reduced systemic perfusion, fluid retention, elevated cardiac filling pressures, decreased coronary perfusion, worsened ischemia, multiorgan failure, worsened myocardial dysfunction, and death. Pressure-volume loops provide a useful construct in visualizing the pathophysiology of CS (Fig. 19.2), as well as the effects of MCS in CS (Fig. 19.3). The rationale for mechanical support in CSMI is to (1) support pump failure and provide increased cardiac output and systemic perfusion, (2) alleviate myocardial ischemia and limit infarct size (after STEMI, a large region of myocardium is often stunned, which may regain function after revascularization with PCI, and MCS can serve to decrease myocardial wall stress and demand while bridging the time for the myocardium to recover function), and (3) delay or halt the "downward spiral." 
Fig. 19.1 "Downward spiral" in cardiogenic shock complicating myocardial infarction. STEMI causes left ventricular dysfunction that begets a "downward spiral" that potentiates itself. Pump failure leads to decreased cardiac output and systemic perfusion, causing increased fluid retention and elevated cardiac filling pressures, decreasing coronary perfusion, and worsening existing myocardial ischemia, which in turn worsens pump function. STEMI ST-elevation myocardial infarction
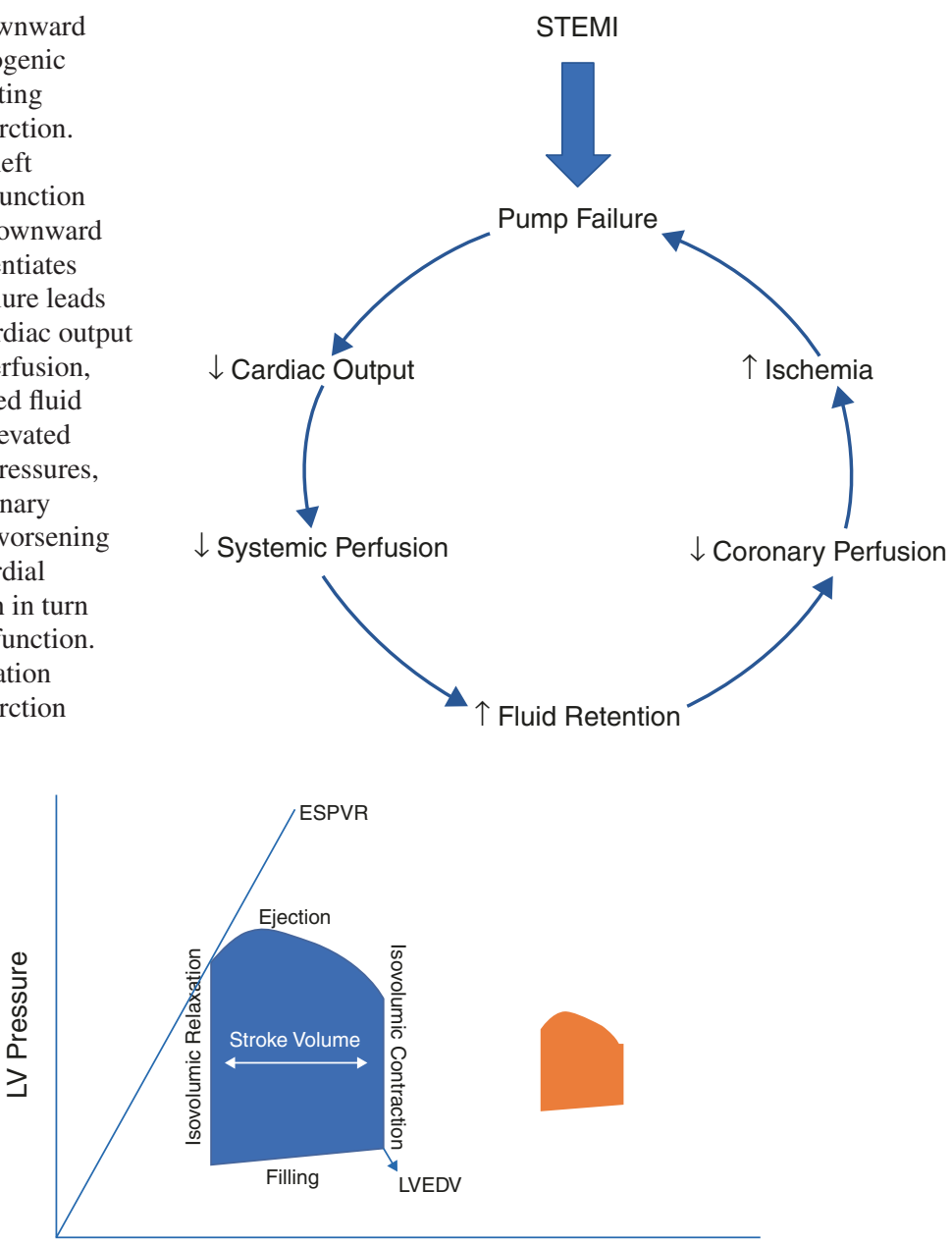

LV Volume

Fig. 19.2 Pressure-volume loops in normal conditions and in cardiogenic shock complicating myocardial infarction. The blue loop represents a pressure-volume loop in normal conditions, and the orange loop represents a pressure-volume loop in CSMI. Stroke volume is represented by the width of the loop, preload is represented by LVEDV, and myocardial contractility is represented by the slope of ESPVR. Compared to normal conditions, CSMI patients have lower stroke volume (decreased width of the loop), higher LVEDV, and reduced contractility (decreased ESPVR slope). $L V$ left ventricle, $L V E D V$ left ventricular end-diastolic volume, ESPVR end-systolic pressurevolume relationship 


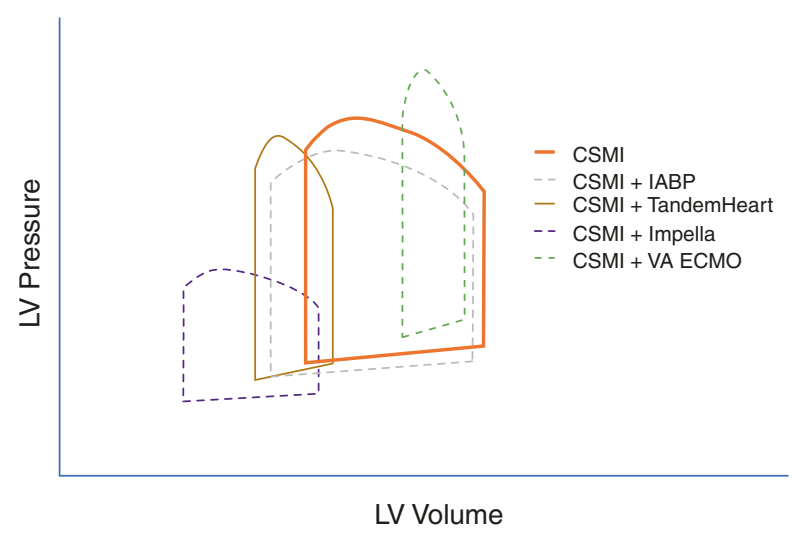

Fig. 19.3 Pressure-volume loops in cardiogenic shock and various mechanical ventricular support devices. The orange loop represents a pressure-volume loop in a patient with CSMI, while the other loops represent pressure-volume loops in patients with CSMI supported by various MCS. IABP reduces LVEDP and increases stroke volume. Both Impella and TandemHeart reduce LVEDP. Notably, VA ECMO increases LVEDP, which increases wall stress and myocardial oxygen demand. $L V$ left ventricle, $C S M I$ cardiogenic shock complicating myocardial infarction, $M C S$ mechanical circulatory support, IABP intra-aortic balloon pump, $L V E D P$ left ventricular enddiastolic pressure, $V A E C M O$ venoarterial extracorporeal membrane oxygenation

\subsection{Intra-aortic Balloon Pump}

\subsubsection{Introduction}

The intra-aortic balloon pump (IABP) is the most widely used mechanical support device (Fig 19.4). The IABP is usually inserted via the femoral artery through a 7.5-8.0 French (Fr) sheath (Table 19.2). It is a polyethylene balloon mounted on a catheter that is inserted into the descending aorta, with the tip ideally positioned $\sim 1-2 \mathrm{~cm}$ distal to the origin of the left subclavian artery. The catheter itself is connected to a console that controls timed inflation of the balloon during the cardiac cycle. Different sizes of the IABP balloon exist and should usually be chosen based on patient height:

- $4^{\prime} 10^{\prime \prime}-5^{\prime} 4^{\prime \prime}$ height should receive $30 \mathrm{~mL}$ IABP balloon.

- $5^{\prime} 4^{\prime \prime}-6^{\prime} 0^{\prime \prime}$ height should receive $40 \mathrm{~mL}$ IABP balloon.

- $>6^{\prime} 0^{\prime \prime}$ height should receive $50 \mathrm{~mL}$ IABP balloon.

\subsubsection{Hemodynamic Effect}

IABP works by counterpulsation in which the balloon is inflated by helium during diastole and deflated during systole. In diastole, inflation of the balloon increases aortic diastolic pressure, which enhances coronary and systemic blood flow. In 
Fig. 19.4 Intra-aortic balloon pump. The intra-aortic balloon pump consists of a polyethylene balloon mounted on a catheter that is inserted into the descending aorta with the tip $\sim 1-2 \mathrm{~cm}$ distal to the origin of the left subclavian artery (Image used with permission from Getinge)

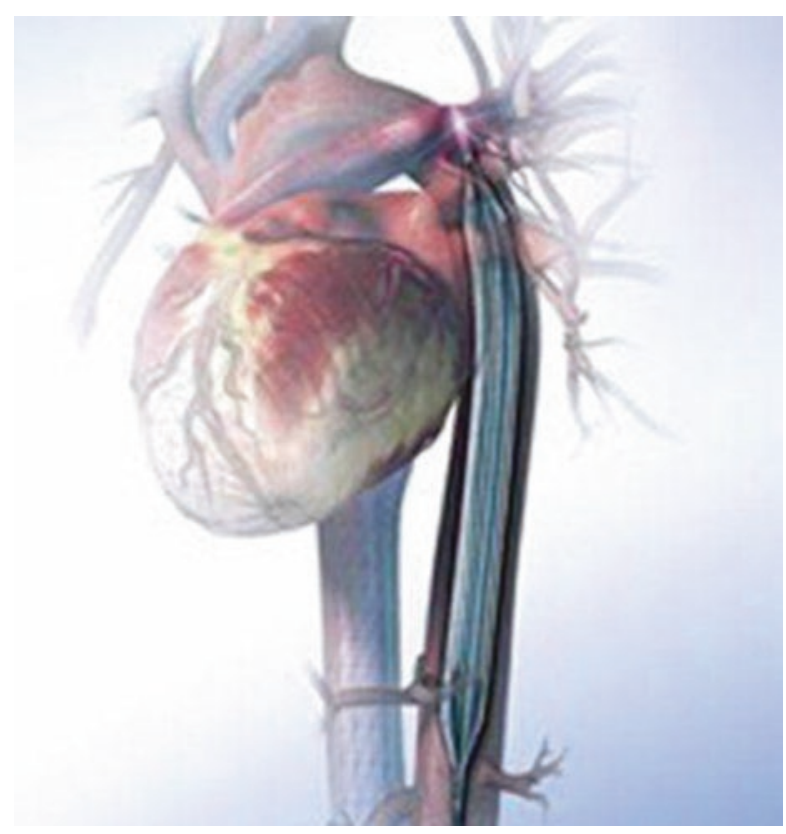

Table 19.2 Comparison of mechanical circulatory support devices

\begin{tabular}{|c|c|c|c|c|}
\hline & IABP & $\begin{array}{l}\text { Impella } 2.5, \mathrm{CP}, \\
5.0\end{array}$ & TandemHeart & VA ECMO \\
\hline Pump mechanism & Pneumatic & Axial & Centrifugal & Centrifugal \\
\hline Sheath size & $7.5-8.0 \mathrm{Fr}$ & $\begin{array}{l}13 \mathrm{Fr}(2.5) ; 14 \mathrm{Fr} \\
(\mathrm{CP}) ; 21 \mathrm{Fr}(5.0)\end{array}$ & $\begin{array}{l}15-19 \mathrm{Fr} \text { arterial; } \\
21 \mathrm{Fr} \text { venous }\end{array}$ & $\begin{array}{l}\text { 15-17 Fr arterial; } \\
18-21 \mathrm{Fr} \text { venous }\end{array}$ \\
\hline $\begin{array}{l}\text { Hemodynamic } \\
\text { support }\end{array}$ & $0.5 \mathrm{~L} / \mathrm{min}$ & $\begin{array}{l}2.5 \mathrm{~L} / \min (2.5) \\
4.0 \mathrm{~L} / \min (4.0) \\
5.0 \mathrm{~L} / \min (5.0)\end{array}$ & $3.5-5.0 \mathrm{~L} / \mathrm{min}$ & $4.0-6.0 \mathrm{~L} / \mathrm{min}$ \\
\hline Afterload & $\downarrow$ & $\downarrow$ & $\uparrow$ & $\uparrow \uparrow \uparrow$ \\
\hline $\begin{array}{l}\text { Risk of limb } \\
\text { ischemia and } \\
\text { bleeding }\end{array}$ & $\uparrow$ & $\uparrow \uparrow$ & $\uparrow \uparrow \uparrow$ & $\uparrow \uparrow \uparrow$ \\
\hline $\begin{array}{l}\text { Complexity of } \\
\text { insertion }\end{array}$ & $\uparrow$ & $\uparrow \uparrow$ & $\uparrow \uparrow \uparrow \uparrow$ & $\uparrow \uparrow \uparrow$ \\
\hline
\end{tabular}

$I A B P$ intra-aortic balloon pump, $V A$ ECMO venoarterial extracorporeal membrane oxygenation, $\mathrm{Fr}$ French

systole, deflation of the balloon reduces left ventricle (LV) afterload and increases cardiac output (Fig. 19.5). The net effect is an increase in mean arterial pressure (MAP), reduction of LV end-diastolic volume and LV end-diastolic pressure, and decreased myocardial oxygen demand (Fig. 19.3). The hemodynamic support provided is $\sim 0.5 \mathrm{~L} / \mathrm{min}$ (Table 19.2). 


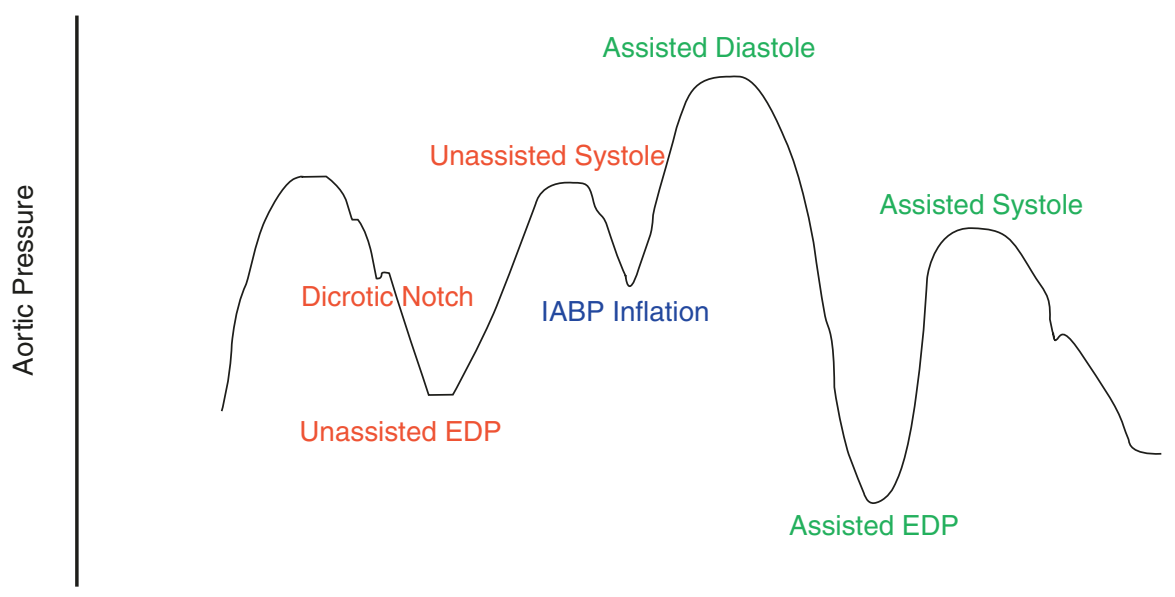

Fig. 19.5 Intra-aortic balloon pump aortic waveform. The IABP inflates at the timing of aortic valve closure or the dicrotic notch on the aortic pressure waveform. Inflation results in diastolic augmentation that is higher pressure than unassisted systole, which results in increased coronary blood flow and MAP. IABP deflates just prior to systole, resulting in a lower assisted aortic EDP and lower assisted systolic pressure. This decrease in afterload decreases myocardial demand and workload. IABP intra-aortic balloon pump, MAP mean arterial pressure, EDP end-diastolic pressure

\subsubsection{Data}

Clinical studies have failed to consistently show benefit of IABP in CSMI. While some retrospective studies and meta-analyses have shown mortality benefit with IABP in CSMI, these studies were often confounded by higher rates of revascularization in patients who received IABP. The most important study of IABP in CSMI was the IABP SHOCK II study published in 2012. This was a multicenter, openlabel trial of 600 patients with CSMI receiving early revascularization who were randomized 1:1 to use of IABP or no IABP. There was no difference in the primary endpoint of 30-day all-cause mortality (39.7\% in IABP group vs. $41.3 \%$ in control group; $p=0.69$ ), and subsequent studies of the cohort failed to show differences in 6- and 12-month mortality. Furthermore, no difference was seen in subgroup analysis or secondary safety endpoints such as bleeding, peripheral complications, sepsis, and stroke. Critics of the study note that $10 \%$ of patients from the control group crossed over to the IABP group, some of the control group patients received left ventricular assist devices, and the vast majority of the patients received the IABP after PCI. Proponents of IABP in CSMI claim that IABP placement may be most beneficial prior to PCI to halt the progression of the "downward spiral" and to reduce infarct size. However, the CRISP AMI (Counterpulsation to Reduce Infarct Size Pre-PCI Acute Myocardial Infarction) multicenter, randomized trial failed to show that the use of IABP prior to PCI reduced infarct size measured by MRI in patients presenting with anterior STEMI without CS. The secondary endpoint of all-cause mortality at 6 months was also not statistically different between groups. 


\subsubsection{Guidelines}

The ACC/AHA guidelines give a Class IIa (level of evidence [LOE] B) recommendation for the use of IABP in CSMI who do not stabilize with pharmacologic therapy. ESC guidelines are more stringent in their recommendation for the use of IABP in STEMI, giving a Class IIa (LOE C) recommendation only in CSMI patients who have a mechanical complication, such as papillary muscle rupture with severe mitral regurgitation or ventricular septal defect. Otherwise, ESC guidelines give IABP a Class III recommendation (LOE B) for routine use in STEMI patients (Table 19.3).

\subsubsection{Contraindications}

- Severe aortic regurgitation

- Aortic dissection

- Severe aortic-iliac-femoral vascular disease

- Severe thrombocytopenia

- Abdominal aortic aneurysm

- Sepsis

\subsubsection{Complications}

The incidence of complications is $\sim 7 \%$ based on outcomes data from the Benchmark Counterpulsation Outcomes Registry of 16,909 patients undergoing IABP therapy between 1996 and 2000. Independent risk factors for major complications of IABP

Table 19.3 ACC/AHA and ESC guidelines for use of mechanical support in ST-elevation myocardial infarction

\begin{tabular}{|c|c|c|c|c|}
\hline \multirow{3}{*}{$\begin{array}{l}\text { Mechanical assist } \\
\text { device }\end{array}$} & \multicolumn{2}{|c|}{ ACC/AHA guidelines } & \multicolumn{2}{|c|}{ ESC guidelines } \\
\hline & \multirow[t]{2}{*}{$\begin{array}{l}\text { Class } \\
\text { IIa } \\
\text { (LOE } \\
\text { B) }\end{array}$} & \multirow[t]{2}{*}{$\begin{array}{l}\text { CSMI that does not } \\
\text { stabilize quickly with } \\
\text { pharmacological therapy }\end{array}$} & $\begin{array}{l}\text { Class } \\
\text { IIa } \\
\text { (LOE } \\
\text { C) }\end{array}$ & $\begin{array}{l}\text { CSMI due to } \\
\text { mechanical } \\
\text { complications }\end{array}$ \\
\hline & & & $\begin{array}{l}\text { Class } \\
\text { III } \\
\text { (LOE } \\
\text { B) }\end{array}$ & Routine use in STEMI \\
\hline $\begin{array}{l}\text { Impella/ } \\
\text { TandemHeart/ } \\
\text { ECMO }\end{array}$ & $\begin{array}{l}\text { Class } \\
\text { IIb } \\
\text { (LOE } \\
\text { C) }\end{array}$ & $\begin{array}{l}\text { May be considered in } \\
\text { refractory CSMI }\end{array}$ & $\begin{array}{l}\text { Class } \\
\text { IIb } \\
\text { (LOE } \\
\text { C) }\end{array}$ & $\begin{array}{l}\text { Short-term use may be } \\
\text { considered in } \\
\text { refractory CSMI }\end{array}$ \\
\hline
\end{tabular}

ACC/AHA American College of Cardiology/American Heart Association, ESC European Society of Cardiology, IABP intra-aortic balloon pump, $L O E$ level of evidence, CSMI cardiogenic shock complicating myocardial infarction, STEMI ST-elevation myocardial infarction, ECMO extracorporeal membrane oxygenation 
were peripheral vascular disease, female sex, body surface area $<1.65 \mathrm{~m}^{2}$, and age $\geq 75$ years. Complications of IABP include:

- Severe bleeding

- Limb ischemia

- Stroke

- Balloon leak or rupture

- Thrombocytopenia

- Hemolysis

- Aortic dissection

- Renal artery, mesenteric artery, or left subclavian artery obstruction

- Infection/sepsis

\subsection{Axial-Flow Pump (Impella)}

\subsubsection{Introduction}

The Impella consists of a miniature axial-flow pump system that continuously pulls blood from the LV into the ascending aorta, thus augmenting cardiac output (Fig. 19.6). The pump is mounted on a catheter that is inserted retrograde across

Fig. 19.6 Impella CP. The Impella is placed retrograde across the aortic valve, with the pigtail and inlet area in the left ventricle and the outlet area in the ascending aorta. Blood is continuously pulled from the left ventricle into the ascending aorta. $C P$ cardiac power (C) 2018 Abiomed, Inc, image used with permission)

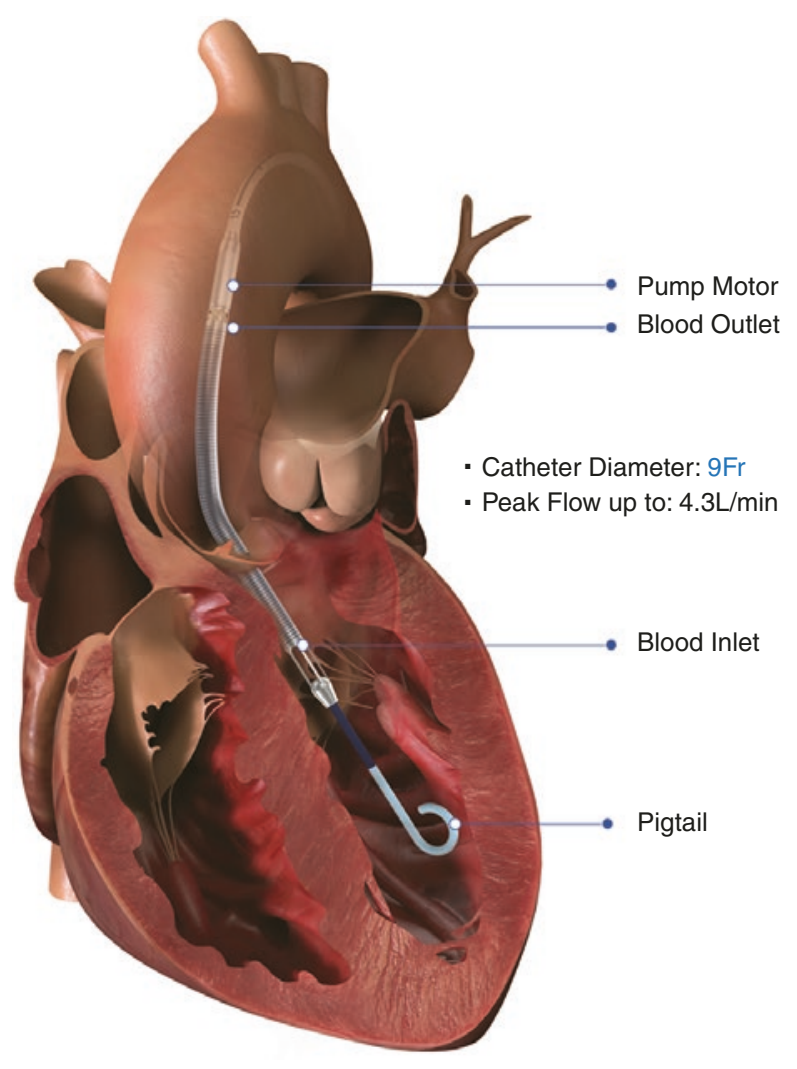


the aortic valve, usually via the femoral artery. The tip of the catheter is in the shape of a pigtail for stabilization and safety of the device in the LV. Near the tip of the catheter is an inlet area, from which blood is pulled to be expelled through the outlet area into the aorta. While three versions of the device are available, Impella 2.5 and the Impella cardiac power (CP) are most commonly used in STEMI since they can both be inserted percutaneously. The third version, Impella 5.0, requires surgical cutdown. Although all three devices have a 9 Fr catheter, the size of the femoral artery sheath varies due to different sizes of the pump motor (Table 19.2):

- Impella 2.5: 12 Fr pump motor requiring a 13 Fr femoral arterial sheath

- Impella CP: 14 Fr pump motor requiring a 14 Fr femoral arterial sheath

- Impella 5.0: 21 Fr pump motor requiring a 22 Fr femoral arterial sheath

Similar to the IABP, the Impella is connected to a console outside of the body that powers and regulates its function.

\subsubsection{Hemodynamic Effect}

By directly removing blood from the LV, the Impella reduces LV end-diastolic volume and end-diastolic pressure. The blood is expelled into the ascending aorta, which increases aortic root pressure and increases coronary perfusion during diastole. The net effect is an increase in cardiac output, MAP, and coronary perfusion, with a decrease in wall stress. The Impella more markedly reduces LV volumes and preload compared with an IABP, thus reducing myocardial oxygen demands of the LV which can be visualized as the area inside the loop (Fig. 19.3). It is also important to note that the width of the pressure-volume loop only shows the stroke volume produced by the heart and not by the contributions of the Impella. Hemodynamic support is $\sim 2.5 \mathrm{~L} / \mathrm{min}$ for the Impella $2.5, \sim 4.0 \mathrm{~L} / \mathrm{min}$ for the Impella $\mathrm{CP}$, and $\sim 5.0 \mathrm{~L} / \mathrm{min}$ for the Impella 5.0 (Table 19.2).

\subsubsection{Data}

The most important study evaluating the use of Impella in CSMI is the ISAR-SHOCK (Efficacy Study of LV Assist Device to Treat Patients with Cardiogenic Shock) trial, which tested whether Impella 2.5 provided superior hemodynamic support in CSMI compared with IABP. This prospective trial randomized 26 patients presenting with CSMI to either IABP or Impella 2.5 implantation after PCI. The primary endpoint of hemodynamic improvement after 30 minutes of device implantation was significantly better in patients receiving the Impella 2.5 versus IABP $(\Delta$ cardiac index $=0.49 \pm 0.46 \mathrm{~L} /$ $\mathrm{min} / \mathrm{m}^{2}$ vs. $0.11 \pm 0.31 \mathrm{~L} / \mathrm{min} / \mathrm{m}^{2} ; p=0.02$ ). Overall 30 -day mortality was $46 \%$ in both groups. However, the study had a small number of patients and was not powered to detect mortality differences between groups. Similar to criticisms of the IABP SHOCK II trial, proponents of Impella 2.5 in CSMI note that the patients in 
ISAR-SHOCK received the mechanical devices only after revascularization, when the expected benefit may not be as large. Indeed, an observational study evaluating 154 consecutive unselected patients from the USpella Registry who received Impella 2.5 in CSMI found that the patients who received Impella 2.5 prior to PCI had significantly better survival to hospital discharge compared with patients who received Impella 2.5 after PCI (65.1 vs. 40.7\%; $p=0.003)$. In the interval following the publication of the ISAR-SHOCK results, the Impella CP became available, which can also be placed percutaneously and should provide greater hemodynamic support in CSMI. However, no randomized trials have evaluated the Impella CP or 5.0 in CSMI.

\subsubsection{Guidelines}

Both ACC/AHA and ESC guidelines give Impella a Class IIb (LOE C) indication for use in refractory CSMI (Table 19.3).

\subsubsection{Contraindications}

- Mechanical aortic valve

- Critical severe aortic stenosis

- Moderate to severe aortic regurgitation

- LV thrombus

- Ventricular septal defect

- Severe peripheral arterial disease

- Severe sepsis

\subsubsection{Complications}

The most common complication of Impella is bleeding, given the need for anticoagulation and the 13-22 Fr size catheters needed for arterial access. In the ImpellaEUROSHOCK-registry that retrospectively evaluated 120 patients receiving Impella 2.5 for CSMI, bleeding requiring transfusion occurred in $24.2 \%$ of patients, and bleeding requiring surgery occurred in $4.2 \%$ of patients. Other complications of Impella include:

- Limb ischemia

- Stroke

- LV injury or perforation

- Cardiac tamponade

- Hemolysis

- Arrhythmias

- Acquired von Willebrand syndrome

- Mitral regurgitation secondary to chordal rupture

- Functional mitral stenosis 


\subsection{Extracorporeal Left Heart Bypass (TandemHeart)}

\subsubsection{Introduction}

The TandemHeart is an extracorporeal left atrial to arterial assist device (Fig. 19.7). Unlike the IABP and Impella that require only arterial access, the TandemHeart requires both venous and arterial access. It is inserted by first creating a transseptal puncture from the right atrium into the left atrium. After a guidewire is passed into the left atrium and the transseptal opening is sufficiently dilated, a $21 \mathrm{Fr}$ cannula is inserted via the femoral vein, up the inferior vena cava, through the right atrium, and into the left atrium. The cannula has a large end-hole and multiple side holes that

Fig. 19.7 TandemHeart. The TandemHeart is a left atrial to arterial extracorporeal device that requires atrial transseptal puncture. A cannula is situated into the left atrium and aspirates oxygenated blood, which is delivered to the body by a femoral artery outflow cannula (Image used with permission from CardiacAssist)

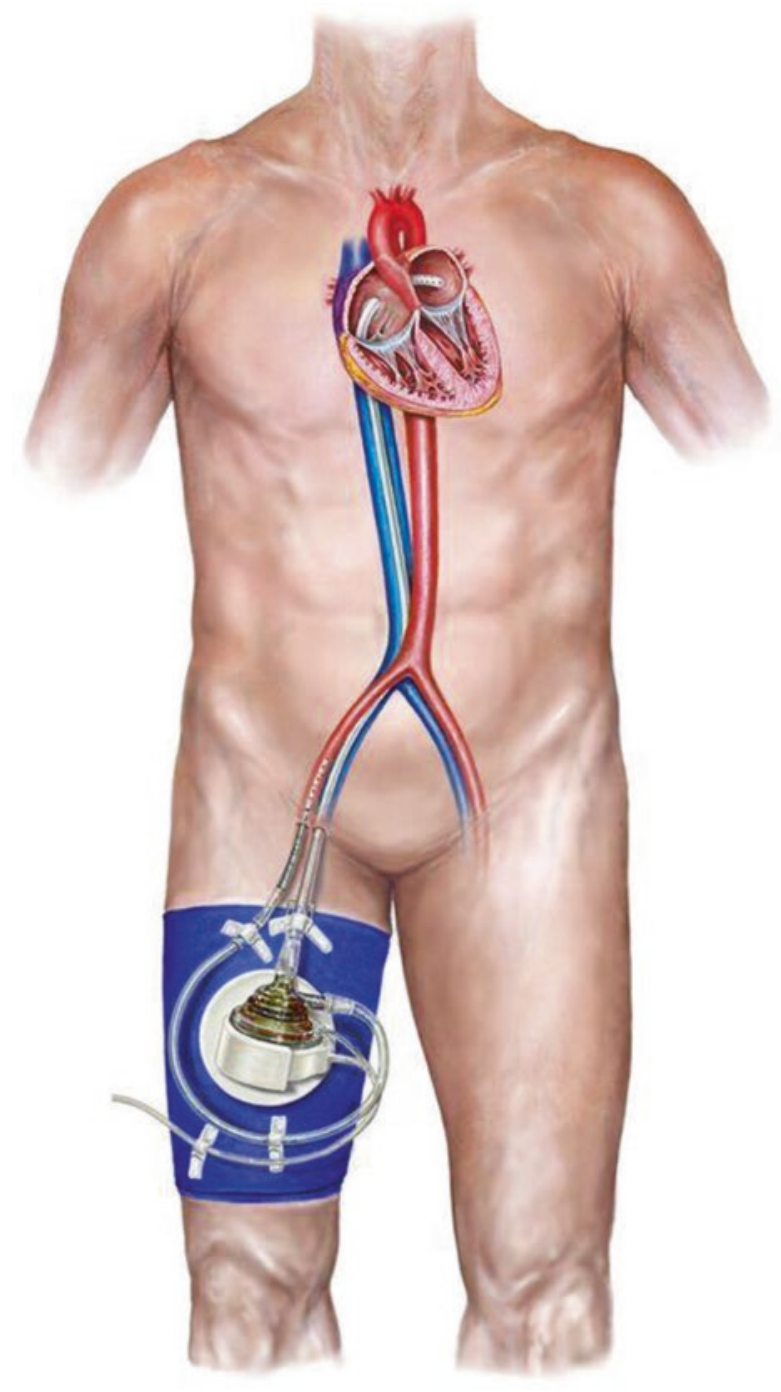


allow for aspiration of oxygenated left atrial blood. The aspirated blood is pulled into an extracorporeal centrifugal pump that pumps the blood into a $15 \mathrm{Fr}-19 \mathrm{Fr}$ arterial outflow cannula in the femoral artery. An external console controls the pump.

\subsubsection{Hemodynamic Effect}

By taking blood from the left atrium, the TandemHeart directly reduces left atrial pressure, which reduces LV end-diastolic volume and LV end-diastolic pressure (Fig. 19.3). Afterload is mildly increased due to the blood flow into the large outflow cannula in the femoral artery. The net effect is an increase in cardiac output, MAP, and coronary perfusion, with a decrease in wall stress. The TandemHeart works in "tandem" with the native heart; therefore, the aorta is still perfused via the native LV pump as well as the TandemHeart pump. However, due to the decrease in preload and increase in afterload associated with the TandemHeart, the contribution to cardiac output from the native heart usually falls. It is not uncommon to have a flat, non-pulsatile arterial waveform on the display console showing little contribution of pulsatile blood flow from heart. Hemodynamic support is $\sim 3.5-5.0 \mathrm{~L} / \mathrm{min}$, mostly depending on the size of the femoral artery outflow cannula (Table 19.2).

\subsubsection{Data}

The most important study evaluating TandemHeart in CSMI was a prospective, single-center, randomized trial published in 2005 comparing IABP to TandemHeart in the setting of intended PCI of the infarct artery in 41 patients. The primary outcome of improvement in cardiac power index was much greater for the TandemHeart group compared with the IABP group $\left(0.22-0.37 \mathrm{~W} / \mathrm{m}^{2}\right.$ vs. $\left.0.22-0.28 \mathrm{~W} / \mathrm{m}^{2} ; p=0.004\right)$. Pulmonary capillary wedge pressure and serum creatinine levels also decreased. However, 30-day mortality was similar between the two groups (IABP $45 \%$ vs. TandemHeart 43\%; $p=0.86$ ). Complications such as severe bleeding and limb ischemia were seen much more frequently in the TandemHeart patients. Limitations of the study included its lack of power to detect mortality differences as well as the lack of generalizability since $>50 \%$ of study patients were excluded from the study.

\subsubsection{Guidelines}

Both ACC/AHA and ESC guidelines give TandemHeart a Class IIb (LOE C) indication for use in refractory CSMI (Table 19.3).

\subsubsection{Contraindications}

- Bleeding diathesis

- Severe peripheral vascular disease 
- Severe right ventricular dysfunction

- Severe aortic regurgitation

- Ventricular septal defect

\subsubsection{Complications}

The major complications of the TandemHeart are severe bleeding and limb ischemia. In the randomized trial in 2005 noted above, 19 of the 21 patients randomized to TandemHeart required blood transfusions. Most of the patients had bleeding at the arterial access site. Seven of the 21 patients developed limb ischemia that either required surgical or percutaneous revascularization. Other complications of TandemHeart include:

- Atrial perforation

- Cardiac tamponade

- Thromboembolism

- Infection/sepsis/systemic inflammatory response syndrome

- Stroke

\subsection{Extracorporeal Membrane Oxygenation}

\subsubsection{Introduction}

Venoarterial extracorporeal membrane oxygenation (VA ECMO) can be percutaneously placed at the bedside or catheterization laboratory table, providing not only circulatory support but also gas exchange (Fig. 19.8). An 18-21 Fr inflow cannula is placed via the femoral vein into the right atrium. A 15-17 Fr outflow cannula is placed via the femoral artery into the aorta (Table 19.2). Deoxygenated blood is removed from the right atrium by an extracorporeal centrifugal pump into a membrane oxygenator for gas exchange, and the oxygenated blood is then pumped back into the patient via the femoral artery cannula. A perfusionist is needed to assemble and manage the circuit.

\subsubsection{Hemodynamic Effect}

Although VA ECMO directly removes blood from the right atrium, it does not decrease LV preload. This is due to pulmonary and bronchial venous return to the LV. VA ECMO also increases afterload due to the retrograde flow via the femoral artery cannula into the aorta. The combined effect of both increased preload and afterload of the LV results in an overloaded LV with elevated LV end-diastolic volume and LV end-diastolic pressure (Fig. 19.3), causing increased wall stress and myocardial oxygen consumption. In CSMI, this increase in wall stress can worsen ischemia and LV function, as well as decrease the likelihood of stunned 


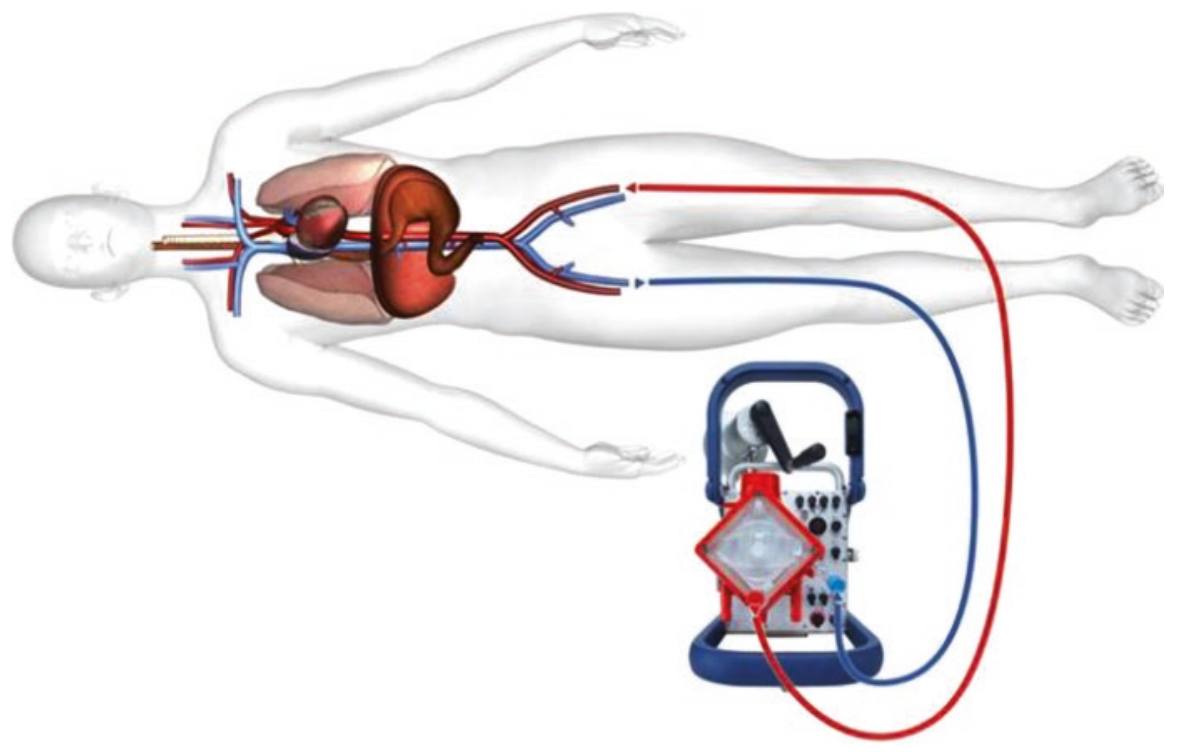

Fig. 19.8 Venoarterial extracorporeal membrane oxygenation. Venoarterial extracorporeal membrane oxygenation is a unique MCS device that provides both circulatory support and gas exchange. Deoxygenated blood is removed from the right atrium by a large venous cannula and pumped through a membrane oxygenator for gas exchange, and the oxygenated blood is delivered to the body through a large outflow femoral artery cannula. MCS mechanical circulatory support (Image used with permission from Getinge)

myocardium to recover. Hemodynamic support is $~ 4.0-6.0 \mathrm{~L} / \mathrm{min}$, with significant increases in MAP and total cardiac output (Table 19.2).

\subsubsection{Data}

The data for ECMO in CSMI are from retrospective, small studies. One singlecenter study evaluated 30-day outcomes in patients with STEMI complicated by profound CS, defined as systolic blood pressure $\leq 75 \mathrm{mmHg}$ after IABP and inotrope agents. This study compared 46 patients with profound CSMI treated with primary PCI and ECMO between 2002 and 2009 with a historical control group of 25 patients with profound CSMI treated with primary PCI without ECMO between 1993 and 2002. Thirty-day mortality in the group treated with ECMO and PCI was significantly lower than the historical group (72 vs. 32\%, RRR 46\%; $p=0.003$ ). However, results may be confounded due to the large temporal differences between the groups, as some of the improvement in mortality may be due to improved adjunctive pharmacotherapy, equipment, and technique. Other retrospective studies have suggested that VA ECMO may be particularly beneficial in CSMI patients with cardiac arrest, with improvements in short- and mid-term survival. No randomized controlled trials exist for ECMO in CSMI. 


\subsubsection{Guidelines}

Both ACC/AHA and ESC guidelines give ECMO a Class IIb (LOE C) indication for use in refractory CSMI (Table 19.3). The ESC guidelines also recommend considering ECMO in CSMI patients who continue to deteriorate despite IABP.

\subsubsection{Contraindications}

- Bleeding diathesis

- Severe peripheral vascular disease

- Prolonged cardiopulmonary resuscitation (CPR)

- Severe aortic regurgitation

\subsubsection{Complications}

As with TandemHeart, severe bleeding and limb ischemia are major complications of ECMO. Unique to ECMO among the mechanical support devices, however, are the complications resulting from LV overload. Over time, LV dilatation, LV thrombus, pulmonary edema, and pulmonary venous hypertension may occur. Strategies to "vent" the LV such as IABP, Impella, atrial septostomy, or direct LV decompression may decrease complications from LV overload. Other complications of ECMO include:

- Acquired von Willebrand deficiency

- Hemolysis

- Thrombocytopenia

- Infection/sepsis/systemic inflammatory response syndrome

- Coronary and cerebral hypoxia

- Renal insufficiency

- Stroke

\subsection{Practical Approach to the Use of Mechanical Support Devices in STEMI}

The main decision points for use of mechanical support in STEMI are (1) when to initiate MCS and (2) which device to use. MCS needs to be carefully selected in STEMI patients. First, an evaluation based on vital signs, physical examination, and available data needs to be conducted. If the patient exhibits no evidence of cardiogenic shock, then prompt revascularization without planned MCS should be the initial strategy. An algorithm for MCS in STEMI is shown in Fig. 19.9.

For patients who initially present in shock, either immediate revascularization or early use of MCS with prompt revascularization is the preferred strategy. As 


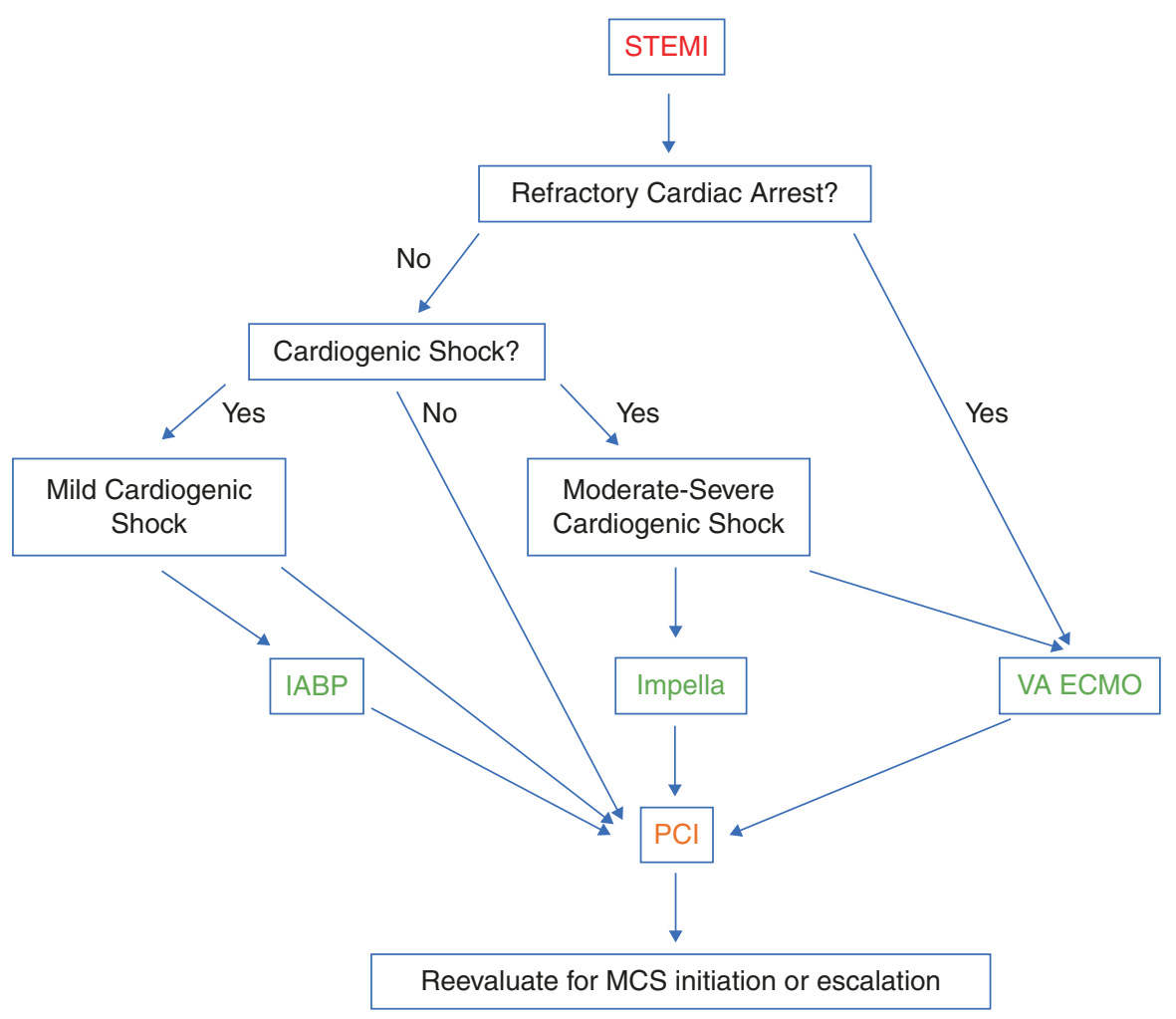

Fig. 19.9 Algorithm for mechanical circulatory support in STEMI. STEMI ST-elevation myocardial infarction, $I A B P$ intra-aortic balloon pump, VA ECMO venoarterial extracorporeal membrane oxygenation, $P C I$ percutaneous coronary intervention

discussed earlier in this chapter, there is no clear evidence that MCS should be routinely used in CSMI. Despite the benefits of MCS, the use of support devices exposes the patient to more complications_especially bleeding—and may also delay door-to-device time. Prompt revascularization in CSMI continues to be the ultimate goal, with Class I indications from both AHA and ESC. The randomized SHOCK trial in 1999 showed that patients presenting with CSMI had statistically significant improved survival at 6 months with emergency revascularization compared with initial medical stabilization, despite a high use of IABP in both groups. Regardless of the initial strategy chosen, once stabilized in the emergency department, patients with CSMI should be immediately rushed to the cardiac catheterization laboratory, at which point the operator can proceed with either primary PCI or MCS with subsequent PCI. If the patient cannot be stabilized in the emergency department and has refractory cardiac arrest despite high-quality advanced cardiovascular life support, then consideration should be given for ECMO.

If the patient with CSMI requires no inotropic or vasopressor therapy, or only low-dose therapy, and signs of only mild CS (wedge $<20$, normal mental status), 
then we generally proceed with immediate PCI. However, if the patient is requiring either increasing doses of adjunctive pharmacologic therapy or is already on multiple inotropes or vasopressors and has signs of moderate to severe CS (wedge > 20, altered mental status, cool extremities, elevated serum lactate), then MCS should first be initiated prior to PCI in the cardiac catheterization laboratory. Pharmacological therapy such as inotropes and vasopressors may be used to stabilize the patient, but these agents may actually be harmful over time. Vasopressors increase MAP and improve hypotension but at the cost of increased LV afterload, which increases myocardial work and demand, leading to worsened myocardial ischemia. Similarly, inotropes improve pump function but directly increase myocardial work and demand, again leading to worsened myocardial ischemia. Furthermore, these drugs are often proarrhythmic and inadequately support a failing heart pump. Thus, while a patient may initially be "stable" on vasopressor and inotrope therapy, the increased myocardial workload and ischemia with these agents may hasten the "downward spiral" of cardiogenic shock. Conversely, mechanical ventricular support devices can provide greater circulatory support, reduce filling pressures, reduce myocardial wall stress and demand, increase coronary perfusion, and even limit infarct size. Therefore, for patients in moderate to severe CS with moderate or high doses of inotropes or vasopressors, MCS prior to PCI should be strongly considered to support the patient and to relieve myocardial demand and ischemia. Similarly, if a STEMI patient who initially received planned PCI without MCS deteriorates and develops CS, then strong consideration should be given for immediate MCS implantation.

Consideration of which mechanical support device to be used is mostly based on the amount of hemodynamic support needed, risk of bleeding, ease and rapidity of placement, and contraindications (Table 19.2). Immediate measurement of LV enddiastolic pressure and the performance of a left ventriculogram should be done to help assess the severity of CS, dysfunction of the LV, and any possible mechanical complications. Additionally, if concern for peripheral arterial disease exists, iliofemoral angiography should be quickly done via a femoral artery sheath to help determine whether the femoral artery is suitable for an MCS device. In general, the favored MCS devices should be the IABP and Impella CP. While the IABP is relatively inexpensive, generally safe, and straightforward to implant, the tradeoff is that it provides only modest hemodynamic support. Impella CP provides much greater hemodynamic support and is a better choice for patients in severe CS. It can still be inserted percutaneously, and provides greater hemodynamic support than the Impella 2.5 , with a femoral artery sheath only 1 Fr size larger. It can provide nearly the same forward cardiac output as the TandemHeart without the technical challenges associated with the latter device (the larger femoral artery sheath and the need for a transseptal puncture). Finally, while VA ECMO can effectively provide complete cardiopulmonary support, it also increases afterload and can worsen myocardial ischemia. Thus, generally patients in mild CS should receive IABP with frequent reevaluation for device escalation (replacement with Impella or VA ECMO), and those with moderate to severe CS should receive Impella CP. VA ECMO should be reserved for those patients in cardiac arrest. 


\subsection{Summary}

MCS in STEMI can be useful in carefully selected patients with cardiogenic shock. Early identification of CS is paramount, and early initiation of MCS can help support a failing LV, reduce filling pressures, improve coronary perfusion, relieve myocardial ischemia, and ultimately prevent the "downward spiral" of CS that leads to multiorgan failure and death. An algorithm for use of MCS in STEMI patients helps select which patients may benefit from MCS and which ventricular support device may be optimal. Ultimately, a multidisciplinary team approach to patients with CSMI is vital, as quick action and communication from EMS, emergency medicine physicians, interventional cardiologists, cardiac surgeons, nurses, and technicians can stabilize and identify the patients who are best candidates for mechanical ventricular support devices.

\subsection{Case Report: Severe Cardiogenic Shock in Context of Cardiac Arrest}

Nathan Lo and E. Magnus Ohman

\subsubsection{Case Report}

A 66-year-old female with history of hypertension, hyperlipidemia, and smoking abuse presented with chest pain at home prompting a call to emergency medical services (EMS). Upon EMS arrival, she was hypotensive with electrocardiogram (ECG) showing sinus bradycardia and ST-elevations in leads II, III, and AVF. On arrival to the emergency department, she sustained a pulseless electrical activity (PEA) arrest requiring CPR, epinephrine administration, and intubation. She regained return of spontaneous circulation (ROSC) after several minutes, but she remained hypotensive requiring norepinephrine drip at a low dose. Physical examination was notable for elevated JVP of $\sim 16 \mathrm{mmHg}, \mathrm{S} 3$ gallop, and no rales. She was given IV heparin and PO aspirin via an orogastric tube. She was quickly taken to the cardiac catheterization laboratory.

On the catheterization laboratory table, she had a bradycardic cardiac arrest requiring CPR, atropine, and epinephrine. She regained ROSC after several minutes. We obtained access in the right femoral artery with a 6 Fr sheath and performed diagnostic angiograms of the left and right coronary arteries. This revealed three-vessel disease with a culprit proximal 95\% dominant RCA thrombotic occlusion, $80 \%$ mid-LAD lesion, and 90\% large ramus lesion (Fig. 19.10). A pigtail catheter was used to cross the aortic valve revealing LVEDP of $19 \mathrm{mmHg}$ and akinetic inferior and apical walls with LV ejection fraction $\sim 40 \%$ on left ventriculogram. Arterial blood gas showed a $\mathrm{pH}$ of $7.07, \mathrm{PCO}_{2}$ of 63 , and $\mathrm{PO}_{2}$ of 154 , and the tidal volume and respiratory rate were increased on the ventilator. 

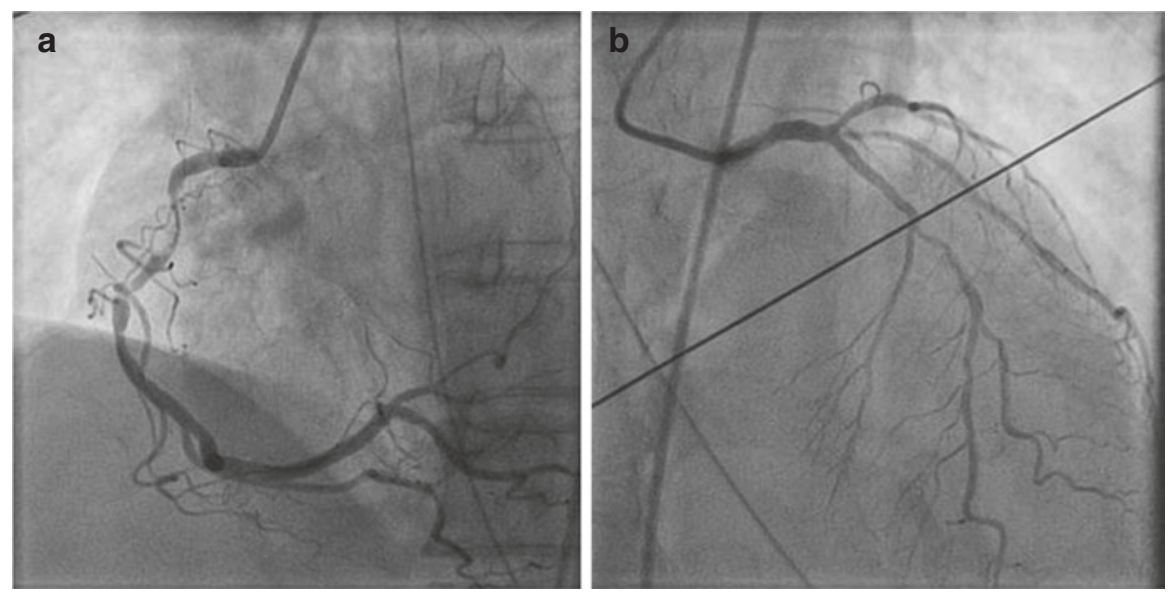

Fig. 19.10 Severe three-vessel disease in a patient with cardiogenic shock complicating STEMI. Angiography reveals a dominant RCA with culprit 95\% lesion (a), 80\% mid-LAD lesion, and $90 \%$ ostial ramus lesion (b). STEMI ST-elevation myocardial infarction, $R C A$ right coronary artery, $L A D$ left anterior descending artery

We decided to proceed directly with PCI of her culprit RCA lesion without upfront MCS due to her mild cardiogenic shock and low vasopressor requirements, although upfront IABP placement could arguably have been better. Eptifibatide bolus and drip was started. On balloon inflation of her RCA occlusion, the patient became bradycardic and hypotensive, requiring further brief CPR, atropine, and epinephrine administration. Angiography of her RCA showed slow flow. We immediately proceeded to IABP placement via the left femoral artery. She had excellent diastolic augmentation with MAP in the 90s. Flow in her RCA improved, and we proceeded to stent her RCA with a $3.0 \times 38 \mathrm{~mm}$ drug-eluting stent, with distal embolization to a posterolateral branch (Fig. 19.11). Her MAP decreased to 70s despite the IABP and vasopressor support. A Swan-Ganz catheter was placed via her right femoral vein which showed mean RA pressure of $16 \mathrm{mmHg}$, RV pressure of $33 / 15 \mathrm{mmHg}$, PA pressure of $33 / 21 \mathrm{mmHg}$ with mean of $26 \mathrm{mmHg}$, and wedge of $19 \mathrm{mmHg}$. PA saturations were 52 and $54 \%$ in duplicate, and Fick CO was $2.2 \mathrm{~L} /$ min with index of $1.4 \mathrm{~L} / \mathrm{min}-\mathrm{m}^{2}$. We considered MCS device escalation to Impella and further revascularization of her other coronary disease, but given her relative stability, we decided to transfer her to the CCU and closely watch her over the next several hours. Her right femoral artery sheath was left in place in case ECMO was required. Transthoracic echocardiogram showed LVEF of $35 \%$ with akinetic inferior and posterior walls and hypokinetic anterior, lateral, and apical walls. RV was mildly enlarged with moderate global dysfunction. Over the next $36 \mathrm{~h}$, her vasopressors were weaned off, she was extubated, and the IABP removed. Her Fick CO improved to $4.2 \mathrm{~L} / \mathrm{min}$ with index of $2.6 \mathrm{~L} / \mathrm{min}-\mathrm{m}^{2}$. She was discharged home in stable condition with plan for elective revascularization of her LAD and ramus. This case illustrates several important points: (1) MCS can be very effective in conjunction with PCI in the treatment of CSMI; (2) even in cases where MCS is not placed 
Fig. 19.11 RCA after stent placement. A long $3.0 \times 38 \mathrm{~mm}$ drug-eluting stent was placed with excellent angiographic result in the proximal to middle RCA. Due to distal embolization, there was initially loss of flow to a posterolateral branch (arrow). $R C A$ right coronary artery

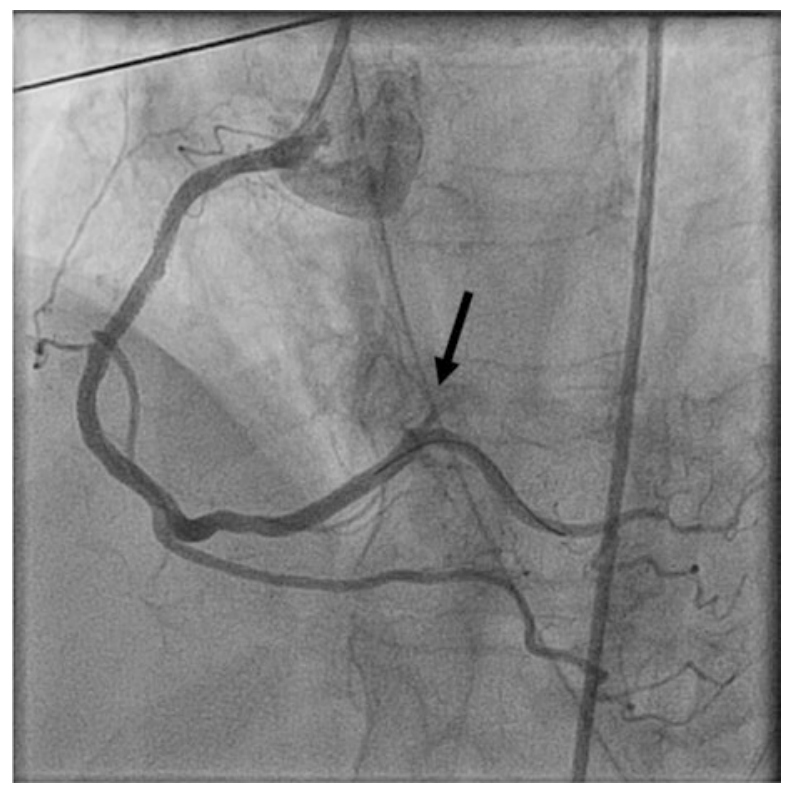

upfront prior to PCI, it is important to always reevaluate whether MCS should be initiated or whether device escalation is necessary; and (3) a clear algorithm can help guide device initiation and selection in CSMI.

\section{Further Readings}

Atkinson TM, Ohman ME, O'Neill WW, et al. A practical approach to mechanical circulatory support in patients undergoing percutaneous coronary intervention. J Am Coll Cardiol Intv. 2016;9:871-83.

Burkhoff D, Sayer G, Doshi D, et al. Hemodynamics of mechanical circulatory support. J Am Coll Cardiol. 2015;66:2663-74.

Doll JA, Ohman EM, Patel MR, et al. A team-based approach to patients in cardiogenic shock. Catheter Cardiovasc Interv. 2016;88(3):424-33.

Ferguson JJ, Cohen M, Freedman RJ, et al. The current practice of intra-aortic balloon counterpulsation: results from the benchmark registry. J Am Coll Cardiol. 2001;38:1456-62.

Ibanez B, James S, Agewall S, et al. 2017 ESC Guidelines for the management of acute myocardial infarction in patients presenting with ST-segment elevation: the task force for the management of acute myocardial infarction in patients presenting with ST-segment elevation of the European Society of Cardiology (ESC). Eur Heart J. 2018;39(2):119-77.

Lauten A, Engstrom AE, Jung C, et al. Percutaneous left-ventricular support with the Impella-2.5assist device in acute cardiogenic shock: results of the Impella-EUROSHOCK-registry. Circ Heart Fail. 2013;6:23-30.

O'Gara PT, Kushner FG, Ascheim DD, et al. 2013 ACCF/AHA Guideline for the management of ST-elevation myocardial infarction. Circulation. 2013;127:e362-425.

Patel M, Smalling R, Thiele $\mathrm{H}$, et al. Intra-aortic balloon counterpulsation and infarct size in patients with acute anterior myocardial infarction without shock: the CRISP AMI randomized trial. JAMA. 2011;306(12):1329-37. 
Rihal CS, Naidu SS, Givertz MM, et al. 2015 SCAI/ACC/HFSA/STS clinical expert consensus statement on the use of percutaneous mechanical circulatory support devices in cardiovascular care. J Am Coll Cardiol. 2015;65:e7-26.

Sayfarth M, Sibbing D, Bauer I, et al. A randomized clinical trial to evaluate the safety and efficacy of a percutaneous left ventricular assist device versus intra-aortic balloon pumping for treatment of cardiogenic shock caused by myocardial infarction. J Am Coll Cardiol. 2008;52:1584-8.

Shah P, Cowger JA. Cardiogenic shock. Crit Care Clin. 2014;30:391-412.

Sheu J, Tsai T, Lee F, et al. Early extracorporeal membrane oxygenator-assisted primary percutaneous coronary intervention improved 30-day clinical outcomes in patients with ST-segment elevation myocardial infarction complicated with profound cardiogenic shock. Crit Care Med. 2010;38:810-1817.

Thiele H, Sick P, Boudiot E, et al. Randomized comparison of intra-aortic balloon support with a percutaneous left ventricular assist device in patients with revascularized acute myocardial infarction complicated by cardiogenic shock. Eur Heart J. 2005;26:1276-83.

Thiele H, Zeymer U, Neumann F, et al. Intra-aortic balloon counterpulsation in acute myocardial infarction complicated by cardiogenic shock (IABP-SHOCK II): final 12 month results of a randomised, open-label trial. Lancet. 2013;382(9905):1638-45.

Thiele H, Zeymer U, Neumann F, et al. Intraaortic balloon support for myocardial infarction with cardiogenic shock. N Engl J Med. 2012;367:1287-96.

Open Access This chapter is licensed under the terms of the Creative Commons Attribution 4.0 International License (http://creativecommons.org/licenses/by/4.0/), which permits use, sharing, adaptation, distribution and reproduction in any medium or format, as long as you give appropriate credit to the original author(s) and the source, provide a link to the Creative Commons license and indicate if changes were made.

The images or other third party material in this chapter are included in the chapter's Creative Commons license, unless indicated otherwise in a credit line to the material. If material is not included in the chapter's Creative Commons license and your intended use is not permitted by statutory regulation or exceeds the permitted use, you will need to obtain permission directly from the copyright holder. 